\title{
ON MINKOWSKI BODIES OF CONSTANT WIDTH
}

\author{
PAUL J. KELLY
}

A metric set is entire if the addition of any point to the set increases the diameter. A convex body has constant width if all pairs of parallel supporting planes are the same distance apart. These concepts are known to be equivalent in euclidean space. ${ }^{1}$ The present paper shows that they are also equivalent in a minkowski space.

A proof for this equivalence for the minkowski plane was given by Meissner. ${ }^{2}$ He showed also that two curves of the same constant width have the same circumference, and that a three-dimensional body of constant width has plane sections of constant width, in terms of the corresponding section of the minkowski sphere as plane indicatrix. However, Meissner's three-space proof for the equivalence of entireness to constant width is incomplete. ${ }^{3} \mathrm{He}$ assumed, moreover, that the indicatrix had no singular points. The equivalence here is shown for the $n$-dimensional case with the assumption merely that the indicatrix is convex.

In a euclidean $E^{n}$ space let $C$ be a closed, convex hypersurface with $O$ as center. In terms of $C$ as indicatrix let a minkowski distance be defined in the usual way to make the space an $M^{n}{ }^{4}{ }^{4}$

Let $r$ and $s$ be half-rays emanating from $O$ at an angle $\theta$ and let $\lambda_{r}, \lambda_{s}$ be the euclidean lengths of the minkowski radii of $C$ in these directions. Then $\operatorname{sm}(r, s)$, a positional sine with respect to $C$, is defined to mean $\lambda_{r} \lambda_{s} \sin \theta_{0}^{5}$

Lemma. If $r, s, t$ are half-rays through $O$, which lie in a plane, with $s$ between $r$ and $t$ (in terms of an angle not greater than $\pi$ ) then $\operatorname{sm}(r, s)$ $+\mathrm{sm}(s, t) \geqq \operatorname{sm}(r, t)$.

If $X_{1}, X_{2}, X_{3}$ are the end points of radii $\lambda_{r}, \lambda_{s}, \lambda_{t}$, then from the convexity of $C$ it follows that the euclidean area of $\Delta O X_{1} X_{2}$ plus the area of $\Delta O x_{2} x_{3}$ is not less than the area of $\Delta O x_{1} x_{3}$. Since the area of

Received by the editors August 16, 1948.

${ }^{1}$ Bonnesen and Fenschel, Theorie der konvexen Körper, Ergebnisse der Mathematik, 1934, p. 128. Jessen, Über konvexe Punktmengen konstanter Breite, Math. Zeit. vol. 29 (1928) pp. 378-380.

2 E. Meissner, Über Punktmengen konstanter Breite, Vierteljahrschrift der Naturforschenden Gesellschaft, 1911.

3 This was already noticed in the references under footnote 1 .

- Bonnesen and Fenschel, cf. footnote 1, p. 23.

- This was taken from a more general minkowski sine function defined by $\mathrm{H}$. Busemann who has in preparation a paper on the subject. 
$\Delta O X_{1} X_{2}$ is $(1 / 2) \operatorname{sm}(r, s)$, and similarly for the other terms, the lemma follows.

THEOREM. If $K$ is a body in $M^{n}$ space which is entire, in terms of the minkowski metric, it is of constant width, in terms of this metric, and conversely.

Proof. (1) Let $K$ be entire. Assume it is not of constant width.

(2) It is easily shown that $K$ is convex and that its diameter is its maximum minkowski width. ${ }^{6}$ Take $O$ interior to $K$ and let $H$ and $E$ be the euclidean supporting functions of $K$ and $C$ ( $C$ plus its interior). If $\xi$ is a unit euclidean vector, the minkowski distance between the supporting planes of $K$ in the direction $\xi$ is given by $W(\xi)=[H(\xi)$ $+H(-\xi)] / E(\xi) .{ }^{7}$ From (1), $W(\xi)$ will assume a maximum and a minimum value.

(3) Let $K^{*}$ be the euclidean polar of $K$ with respect to the unit euclidean sphere ("polar," throughout, will refer to the unit euclidean sphere). To a point $U$ of $K^{*}$ there corresponds a unit euclidean vector $\xi$ in the direction from $O$ to $U$, and a width, as in (2) $W(\xi)$. Points of $K^{*}$ will be called maximal or minimal whenever the corresponding width is maximal or minimal. Since $W(\xi)=W(-\xi)$, to each $U$ of $K^{*}$ there is a counterpoint $U^{\prime}$ on $K^{*}$ in the direction $-\xi$ and to which the same width corresponds.

(4) If $K^{*}$ contains a non-maximal, extreme point, then the assumption in (1) is false. For suppose $U_{0}$ such a point. From continuity, $U_{0}$ has a neighborhood of non-maximal points. Let a plane $\pi$, at a distance $\delta$ from $U_{0}$, cut $K^{*}$ into two parts and let $K_{1}^{*}$ be that part separated from $U_{0}$ by $\pi$. Since $K_{1}^{*}$ is properly contained in $K^{*}$, then $K_{1}$, the polar of $K_{1}^{*}$, properly contains $K$. Since the width of $K_{1}$ differs from that of $K$ only in the neighborhood of a non-maximal direction, it follows from continuity that if this neighborhood, that is, $\delta$, is chosen sufficiently small, then the maximal widths of $K$ and $K_{1}$ will be the same. Hence the diameters will be the same. Since $K_{1}$ properly contains $K$, this contradicts the entireness of $K$ and the assumption in (1) must be false.

(5) If $U_{1}, U_{2}, U_{3}$ are points of $K^{*}$ which are collinear, in that order, and which have collinear counterpoints, then $W\left(\xi_{2}\right)$ $\geqq \min \left[W\left(\xi_{1}\right), W\left(\xi_{3}\right)\right]$. We prove this as follows.

(5.1) Let $C^{*}$ be the polar of $C$. This is again a central, convex body and can be used as indicatrix for a second minkowski metrization of $E^{n}$. Let $\left|u_{i}\right|^{*}$ represent the minkowski distance, with respect

${ }^{6}$ Cf. H. Busemann, Intrinsic area, Ann. of Math. vol. 48 (1947) pp. 234-267.

7 Ibid. 
to $C^{*}$, from $O$ to $U_{i},\left|u_{i}\right|$ the corresponding euclidean distance, $\lambda_{i}$ the euclidean length of the radius of $C^{*}$ in the direction $\xi_{i}$, and $\theta_{i j}$ the angle from $\xi_{i}$ to $\xi_{j}$. By definition, then, $\left|u_{i}\right|=\lambda_{i}\left|u_{i}\right|^{*}$. In this notation, the fact that the euclidean area of $\Delta O u_{1} u_{3}$ equals that of $\Delta O U_{1} U_{2}$ plus that of $\Delta O U_{2} U_{3}$ can be expressed as $\lambda_{1} \lambda_{3}\left|u_{1}\right| *\left|u_{3}\right|^{*} \sin \theta_{13}$ $=\lambda_{1} \lambda_{2}\left|u_{1}\right|^{*}\left|u_{2}\right|^{*} \sin \theta_{12}+\lambda_{2} \lambda_{3}\left|u_{2}\right| *\left|u_{3}\right| * \sin \theta_{23}$. Using the positional sine of the lemma (now with respect to $C^{*}$ ), the above relation can be put in the form

$$
\frac{1}{\left|u_{2}\right|^{*}}=\frac{1}{\left|u_{3}\right|^{*}} \frac{\operatorname{sm}\left(\xi_{1}, \xi_{2}\right)}{\operatorname{sm}\left(\xi_{1}, \xi_{3}\right)}+\frac{1}{\left|u_{1}\right|^{*}} \frac{\operatorname{sm}\left(\xi_{2}, \xi_{3}\right)}{\operatorname{sm}\left(\xi_{1}, \xi_{3}\right)} .
$$

(5.2) Directly, from the definitions of minkowski distance and polar body, we have $\left|u_{i}\right|^{*}=\left[H\left(\xi_{i}\right)\right]^{-1} /\left[E\left(\xi_{i}\right)\right]^{-1}=E\left(\xi_{i}\right) / H\left(\xi_{i}\right), i=1,2,3$. Hence the last relation in (5.1) can be written

$$
\frac{H\left(\xi_{2}\right)}{E\left(\xi_{2}\right)}=\frac{H\left(\xi_{3}\right) \mathrm{sm}\left(\xi_{1}, \xi_{2}\right)}{E\left(\xi_{3}\right) \mathrm{sm}\left(\xi_{1}, \xi_{3}\right)}+\frac{H\left(\xi_{1}\right) \mathrm{sm}\left(\xi_{2}, \xi_{3}\right)}{E\left(\xi_{1}\right) \mathrm{sm}\left(\xi_{1}, \xi_{3}\right)}
$$

(5.3) From the linearity of the counterpoints, $U_{i}^{\prime}$, the same area argument can be applied to the three triangles $O U_{i}^{\prime} U_{j}^{\prime}, i \neq j$. The fact that $C^{*}$ is central, and the equality of vertical angles, allows the conclusion to be put in the form

$$
\frac{H\left(-\xi_{2}\right)}{E\left(\xi_{2}\right)}=\frac{H\left(-\xi_{3}\right) \mathrm{sm}\left(\xi_{1}, \xi_{2}\right)}{E\left(\xi_{3}\right) \mathrm{sm}\left(\xi_{1}, \xi_{3}\right)}+\frac{H\left(-\xi_{1}\right) \mathrm{sm}\left(\xi_{2}, \xi_{3}\right)}{E\left(\xi_{1}\right) \mathrm{sm}\left(\xi_{1}, \xi_{3}\right)}
$$

and this, added to (5.2), gives

$$
W\left(\xi_{2}\right)=\frac{W\left(\xi_{3}\right) \operatorname{sm}\left(\xi_{1}, \xi_{2}\right)}{\operatorname{sm}\left(\xi_{1}, \xi_{3}\right)}+\frac{W\left(\xi_{1}\right) \mathrm{sm}\left(\xi_{2}, \xi_{3}\right)}{\operatorname{sm}\left(\xi_{1}, \xi_{3}\right)}
$$

or

$$
W\left(\xi_{2}\right) \geqq \min \left\{\left[W\left(\xi_{1}\right), W\left(\xi_{3}\right)\right]\right\} \cdot\left[\frac{\mathrm{sm}\left(\xi_{1}, \xi_{2}\right)+\operatorname{sm}\left(\xi_{2}, \xi_{3}\right)}{\operatorname{sm}\left(\xi_{1}, \xi_{3}\right)}\right] .
$$

From the lemma, the last factor above is equal to or greater than one, which establishes (5).

(6) If $K^{*}$ contains no non-maximal, extreme points then the assumption in (1) is false. For suppose every extreme point is also a maximal point. Being convex, $K^{*}$ is the union of all convex simplices whose vertices are extreme points. ${ }^{8}$ Then every nonextreme boundary

\footnotetext{
${ }^{8}$ Bonnesen and Fenschel, cf. footnote 1, p. 9.
} 
point lies on a simplex, of dimension equal to or less than $n-1$, which has maximal vertices. From (5), the edge points of this simplex must be maximal and this, again from (5), implies the face points must be maximal. Hence all the boundary points must be maximal, which is to say that only the maximal width is assumed, or that $K$ is of constant width.

(7) Since (4) or (6) must hold, the assumption in (1) is false, and $K$ is of constant width if it is entire.

(8) The counter equivalence, that if $K$ is of constant width it is entire, is easy to establish and can be argued exactly as in the euclidean case.

University of Southern California 